\title{
A Practical Jointed Approach to Thermal Stress Analysis of FGM Disc
}

\author{
Ayşegül Özgür ${ }^{a}$, Kutay Aydın ${ }^{b}$, Ibrahim Keles ${ }^{c} *$ \\ ${ }^{a}$ Amasya University, Graduate School of Natural and Applied Sciences, Department of Mechanical \\ Engineering, Amasya 05100, Turkey \\ ${ }^{a}$ Amasya University, Faculty of Technology, Department of Mechanical Engineering, Amasya 05100, Turkey \\ ${ }^{b}$ Samsun University, Faculty of Engineering, Department of Mechanical Engineering, Samsun 55080, Turkey \\ ${ }^{*} E$-mail address: aysegulnimet@hotmail.com ${ }^{\text {a }}$, kutay.aydin@amasya.edu.tr ${ }^{b}$, ibrahim.keles@samsun.edu.tr ${ }^{c}$
}

ORCID numbers of author:

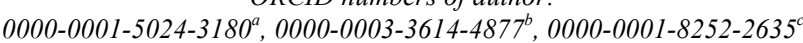

Received date: 12.10 .2020

Accepted date: 06.12.2020

\begin{abstract}
In this study, a numerical approach has been introduced in the elastic stress solutions of hollow disks made of functionally graded materials (FGM) that are exposed to linearly increasing temperature dispersion. The modulus of elasticity and the coefficient of thermal expansion of the FGM disk is assumed to vary in radial direction in different forms, and it is further assumed that the Poisson's ratio is constant. It causes the differential equation that manages the behaviour of the object under different material properties and boundary conditions to be a variable coefficient equation. Except for some simple grade materials and boundary conditions, it is hardly possible to produce an analytical solution of such equations. In this case, the solution of the problems can only be found with numerical approaches. Complementary Functions Method (CFM) was used to solve the problem. Different material models were used from the written works and corresponding radial, tangential and equivalent stresses and radial displacements were calculated. Simple, effective and well-structured solution steps can be easily implemented for disks.
\end{abstract}

Keywords: Functionally Graded Materials, Complementary Functions Method, Hollow Disc, Thermal Stress Analysis.

\section{Introduction}

The issue of the effect of variable thickness FGM cylindrical materials and high temperatures on these structures has become increasingly popular. Applications of cylindrical FGM structures include aerospace, nuclear power plants, aero-marine and chemical plants where the metals and metal alloys used exhibit elastic behavior. FGMs are variegated materials with continuous fluctuation of elastic and thermal properties throughout the material. Constituent materials having various properties are formed by methodically changing the bulk portion of the materials. The materials used in FGM applications are heat resistant, corrosion durable, erosion and elevated breakage toughness. Therefore, the material requirements are quite advanced as these structures are often subjected to high density heat fluxes and are subject to significant changes. Therefore, precise and accurate heat transfer analysis of thick-walled 
cylindrical FGM structures is a requirement for engineering design and production. FGMs were created as updraft protector for aerospace and various reactors as their first application. Nowadays, under the thermomechanical loading, flywheel, turbine, such as high-temperature operating environments, such as components of the machine elements began to be used in general. The use of heterogeneous materials, called FGM, has been increasing recently. FGMs: These are composite materials that consist of two components and have mechanic properties that vary depending on the location in a continuous functional structure. Thermal stress analysis has been performed in structures made of FGM, which are frequently used in engineering structures (such as cylindrical containers, circular discs, pressure vessels, beams and hollow spheres)[1-4]. Although there have been many studies on the analysis of isotropic and laminated composite beams (i.e., [5-9]), however, the research effort dedicated to stability analysis of rectangular of FG plates has been very limited.

The numerical and exact solutions of thermo-elastic analysis of FG rotating disks have submitted by Arnab et al. [10]. Zenkour [11] examined the effect of gradient grading on FG rotating solid disks on radial displacement and stresses in a sandwich structure. Çallioğlu et al. [12] calculated thermal stresses by combining the high level shear deformation theory with multi-layer method in rotating thick-walled cylindrical containers made of FGM. Sharma et al. [13] showed tensile stress and displacement for the thin FGM disk-shaped under the influence of temperature dispersion, angular velocity and thickness. Durodola and Adlington [14] examined the effects of non-homogeneous material parameters on stresses on stress analysis on FG rotary discs (and rotors) at a certain angular velocity. Go et al. [15], using the finite element approach, have shown that a regular cutter or grinding disc with circular free force uniformity can be designed by properly controlling certain parameters to have better thermo-elastic properties. Hassani et al. [16] using Mindlin's theory, created stress distributions on FG rotary discs with nonhomogeneous thickness under thermal loads. Liew et al. [17] analyzed the thermal stresses in the FGM cylinder. Kordkheili and Naghdabadi [18] obtained a quasianalytical resolution analysis with the centrifugal force and power law dispersal of the volume portion under volumetric thermal loads for a fine axial symmetrical rotary free wheel drive made of FGM. Based on Afsar and Go [19], 2-D thermoelastic theories, the FGM rotating disc with radial direction exponentially varying material properties exposed to combined thermal and centrifugal load was investigated. Peng and $\mathrm{Li}$ [20] have developed an efficient method by transforming the thermo-elastic behavior of the FGM disc, whose material properties arbitrarily changing radial way, into Fredholm integral equations. The effects of the gradient considering both the law of power and radial homogeneity on the stress dispersion in FGM rotary solid disks have investigated through Peng and Li [21]. Naghdabadi and Kordkheili [22] obtained using a finite element method for thermo-elastic analysis of FG number plates and crusts. You et al. [23] are utilized a Runge-Kutta mathematical solution technique for elastoplastic stresses on revolving discs of varying thickness and density. A design of the thermo-elastic loaddependent FGM disc designed with load optimization is proposed by Khorsand and Tang [24].

This paper, the evaluation of hollow FGM discs subject to linearly rising temperature dispersion, with the boundary conditions, is reproduced by deriving radial, tangential, equivalent stresses and radial displacement equations. Two different functions form such as power-law, exponential with free state(fr-f) and fixed-free(fx-f) boundary conditions are applied for the governing equation. The differential equations obtained mathematically in the space coordinate system consist mostly of variable coefficients. Thus, this situation indicates the two-point boundary value problem. This article, the complementary functions method (CFM), which is an effective analysis procedure [1, 25-27] as a starting-worth problem, which 
can be resolved by traditional procedures in the current written works, is used. In this study, fourth grade Runga-Kutta (RK4) method was used. The main of the Runge-Kutta method is easy to apply, provides better precision in numerical approximation cases, and process dynamics can be solved efficiently with solid differential equation models. Analytical benchmarking solutions for a homogeneous disk are utilized to verify outcomes and to observe the merging of numerical resolutions. In the current process, the place of the collocation points can be selected randomly. The major aim of this research is to show an effective and correct resolution technique. CFM is an effective and basic resolution procedure with a theoretic background in the written works $[28,29]$ The technique is effectively employed to structures such as curved rods [30], composite beams [31], cylinders [32, 33], spheres [34] and annular fin $[35,36]$ with different structural mechanical problems.

\section{Formulation of Thermal Elastic Solution of the Disk}

The equilibrium differential equation for the plane stress state for thick wall hollow circular disks is expressed in the form below.

$$
r_{i} \leq r \leq r_{o} \quad \frac{d\left(\sigma_{r}\right)}{d r}-\frac{\left(\sigma_{r}-\sigma_{\theta}\right)}{r}=0
$$

where inside radius $r_{i}$, outside radius $r_{o}$, radial stress $\sigma_{r}$ and circumferential stress are $\sigma_{\theta}$. Tangential $\sigma_{r}$ and radial stresses $\sigma_{\theta}$ can be written in terms of Airy stress function $F$

$$
\sigma_{\theta}=\frac{d F}{d r} \quad \sigma_{r}=\frac{F}{r}
$$

The relationships between strains and stresses occurring in an FGM disk in the impact of temperature for elastic materials can be explained by Hooke's law.

$$
\begin{aligned}
& \varepsilon_{r}=\frac{1}{E(r)}\left(\sigma_{r}-v \sigma_{\theta}\right)+\alpha(r) T(r) \\
& \varepsilon_{\theta}=\frac{1}{E(r)}\left(\sigma_{\theta}-v \sigma_{r}\right)+\alpha(r) T(r)
\end{aligned}
$$

where $T(r)$ is the size of the temperature distribution (Eq. 5), $v$ is Poisson's rate, $E(r)$ is elasticity modulus and $\alpha(r)$ is varying thermal growth coefficient. Elasticity modulus and thermal growth coefficient were used as power function (Pwr) (Eq. 6) and as exponential function (Exp) (Eq. 7) of radial direction.

$$
T(r)=T_{0}\left(\frac{r-r_{i}}{r_{o}-r_{i}}\right)
$$




$$
\begin{array}{cc}
\alpha(r)=\alpha_{0}\left(\frac{r}{r_{o}}\right)^{m} & E(r)=E_{0}\left(\frac{r}{r_{o}}\right)^{n} \\
\alpha(r)=\alpha_{0} e^{m r} & E(r)=E_{0} e^{n r}
\end{array}
$$

where $E_{0}$ and $\alpha_{0}$ are nominal elasticity modulus and thermal expansion coefficient, $T_{0}$ is ambient temperature and $n, m$ are equivalent to nil for a homogenous disc. Strain-displacement relation are shown in Eq. 8.

$$
\varepsilon_{\theta}=\frac{u}{r} \quad \varepsilon_{r}=\frac{d u}{d r}
$$

where the radial displacement is represented by $u$. The deformation compatibility equation (Eq. 9) is obtained from Eq. 8.

$$
\varepsilon_{r}=\frac{d}{d r}\left(r \varepsilon_{\theta}\right)
$$

By making use of Eqs. (1), (2), (3) and (4), the equilibrium equations in Eqs. (10a) and (10b) read

$$
\begin{aligned}
& F^{\prime \prime}-F^{\prime}\left(\frac{n-1}{r}\right)-F \frac{(1-n v)}{r^{2}}=\frac{\left(\frac{r}{r_{o}}\right)^{n+m}\left[1+m\left(\frac{r_{i}}{r}-1\right)\right] T_{0} \alpha_{0} E_{0}}{r_{o}-r_{i}} \\
& F^{\prime \prime}-F^{\prime}\left(n-\frac{1}{r}\right)-F(1-n r v)=\frac{(e)^{r(n+m)}\left[m\left(r_{i}-r\right)-1\right] T_{0} \alpha_{0} E_{0}}{r_{o}-r_{i}}
\end{aligned}
$$

Based upon von-Mises failure principle, equivalent stress is described by (Eq. 11)

$$
\sigma_{v m}=\sqrt{\left(\sigma_{r}^{2}-\sigma_{r} \sigma_{\theta}+\sigma_{\theta}^{2}\right)}
$$

The boundary condition is selected as Eq. 12 (fr-f) and Eq. 13 (fx-f) depending on the inside and outside radius of the disk.

$$
\begin{gathered}
\sigma_{\left.r\right|_{r=r_{i}}=} 0 \text { and }\left.\sigma_{r}\right|_{r=r_{O}}=0 \\
\left.u\right|_{r=r_{i}=0}
\end{gathered}
$$




\section{Numerical Solution with CFM}

The constructed of the inhomogeneous governing equations were shown in Eq. 10a and Eq. 10b. Where $F^{\prime}$ expressions denote derivatives taken according to $r$ [31]. The constructed form boundary conditions rewritten in Eq. 12 and Eq. 13.

The solution of (n)th order common differential equations by CFM [1] :

$$
y(x)=y_{o}(x)+b_{1} y_{1}(x)+\ldots+b_{n} y_{n}(x)
$$

where $y_{o}$ is nonhomogeneous resolution and $y_{1} \ldots y_{n}$ are standardized solution.

The general solution of Eq. 10a and Eq. 10b in CFM over the interval $\left[r_{i}, r_{o}\right]$ is given by

$$
\begin{aligned}
& F(r)-F_{0}(r)=b_{1} F_{1}(r)+b_{2} F_{2}(r) \\
& F^{\prime}(r)-F_{0}^{\prime}(r)=b_{1} F_{1}^{\prime}(r)+b_{2} F_{2}^{\prime}(r)
\end{aligned}
$$

The CFM solutions (Eq. 15a and 15b) are calculated from GE's (Eq. 10a and Eq. 10b) using with fourth order Runge-Kutta method (RK4). Mathematical repetitions are produced in the $r_{i} \leq r \leq r_{o}$ variety with the footsteps of $h=0.001$. In RK4 to get the solutions Eq. $15 \mathrm{a}$ and 15b, the boundary worths of governing equations (Eq. 16) are utilized variant transformation as sees

$$
F^{\prime}=Z_{2} \quad F=Z_{1}
$$

As a consequence of RK4, $b_{1}$ and $b_{2}$ can be obtained for fr-f (Eq. 17) and fx-f (Eq. 18) conditions as follows

$$
\begin{gathered}
\left|\begin{array}{ll}
F_{1}\left(r_{i}\right) & F_{2}\left(r_{i}\right) \\
F_{1}\left(r_{o}\right) & F_{2}\left(r_{o}\right)
\end{array}\right| x\left|\begin{array}{l}
b_{1} \\
b_{2}
\end{array}\right|=\left|\begin{array}{l}
-F_{0}\left(r_{i}\right) \\
-F_{0}\left(r_{o}\right)
\end{array}\right| \\
\left|\begin{array}{cc}
r_{i} F_{1}^{\prime}\left(r_{i}\right)-v F_{1}\left(r_{i}\right) & r_{i} F_{2}^{\prime}\left(r_{i}\right)-v F_{2}\left(r_{i}\right) \\
F_{1}\left(r_{o}\right) & F_{2}\left(r_{o}\right)
\end{array}\right| x\left|\begin{array}{l}
b_{1} \\
b_{2}
\end{array}\right|=\left|\begin{array}{c}
v F_{0}\left(r_{i}\right)-r_{b} F_{0}^{\prime}\left(r_{i}\right) \\
-F_{0}\left(r_{o}\right)
\end{array}\right|
\end{gathered}
$$

\section{Confirmation of the Suggested Solution Program}

The analytical solutions obtained using the notations of the current study to a homogeneous disc are given below. In solutions, symbolized by the modulus of elasticity $E$ and Poisson's rate $v$, radial displacement is 
Table 1. Collation of CFM results with analytic solutions for homogeneous disk.

\begin{tabular}{ccccccc}
\hline$r / r_{0}$ & \multicolumn{3}{c}{$U$} & \multicolumn{2}{c}{$\sigma_{r}$} & \multicolumn{2}{c}{$\sigma_{\theta}$} \\
\hline & CFM & Analytic & CFM & Analytic & CFM & Analytic \\
\hline 0.2 & 0.0000000 & 0.0000000 & 0.0000000 & 0.0000000 & -1.0000000 & -1.0000000 \\
0.3 & 0.0001350 & 0.0001350 & -0.2474747 & -0.2474747 & -0.5479797 & -0.5479797 \\
0.4 & 0.0003600 & 0.0003600 & -0.2897727 & -0.2897727 & -0.3011362 & -0.3011362 \\
0.5 & 0.0006750 & 0.0006750 & -0.2727272 & -0.2727272 & -0.1136362 & -0.1136362 \\
0.6 & 0.0010800 & 0.0010800 & -0.2323232 & -0.2323232 & 0.0505052 & 0.0505052 \\
0.7 & 0.0015750 & 0.0015750 & -0.1808905 & -0.1808905 & 0.2036182 & 0.2036182 \\
0.8 & 0.0021600 & 0.0021600 & -0.1235795 & -0.1235795 & 0.3508525 & 0.3508525 \\
0.9 & 0.0028350 & 0.0028350 & -0.0628505 & -0.0628505 & 0.4946690 & 0.4946690 \\
1.0 & 0.0036000 & 0.0036000 & 0.0000000 & 0.0000000 & 0.6363636 & 0.6363636 \\
\hline
\end{tabular}

Radial stress

$$
U=\frac{r}{\left[\left(\frac{r_{0}}{r_{i}}\right)^{2}-1\right]\left[\frac{E(1+v)}{1-v^{2}}\right]}-\frac{r_{0}^{2} / r}{\left[\left(\frac{r_{0}}{r_{i}}\right)^{2}-1\right]\left[\frac{E(v-1)}{1-v^{2}}\right]}
$$

$$
\sigma_{r}=\frac{1-\left(\frac{r_{0}}{r_{i}}\right)^{2}\left(\frac{r}{r_{i}}\right)^{-2}}{\left(\frac{r_{0}}{r_{i}}\right)^{2}-1}
$$

Hoop stress

$$
\sigma_{r}=\frac{1+\left(\frac{r_{0}}{r_{i}}\right)^{2}\left(\frac{r}{r_{i}}\right)^{-2}}{\left(\frac{r_{0}}{r_{i}}\right)^{2}-1}
$$

Table 1 is given in order to compare these results with analytical results by calculating radial and tangential stresses and radial displacement values at 9 points throughout the thickness, provided that they are collocated using the disk sizes and material properties given in Table 2.

The exact results listed in Table 1 reveal the good correctness and efficacy attained by the CFM when analyzing the findings achieved from the above analytic comparison solutions for the homogeneous disk; Calculations made only at 9 points along the thickness gave exact numerical results. 


\section{Numerical Results and Discussion}

A hollow disk made of a FGM and Table 2 shows that disk dimensions and material properties.

Table 2. Disk dimensions and material properties

\begin{tabular}{ccc}
\hline Parameter & Unit & Value \\
\hline$r_{i}$ & $\mathrm{~mm}$ & 20 \\
$r_{o}$ & $\mathrm{~mm}$ & 100 \\
$E_{0}$ & $\mathrm{GPa}$ & 200 \\
$\alpha_{0}$ & $1 /{ }^{\circ} \mathrm{C}$ & $12 \times 10^{-6}$ \\
$T_{0}$ & ${ }^{\circ} \mathrm{C}$ & 300 \\
$v$ & - & 0.29 \\
\hline
\end{tabular}

Figure 1-a illustrates radial stresses in the FGM hollow disk throughout its radius caused by thermal lading for $n=0.5, m=0.5$ used for boundary conditions of both the Pwr and Exp. The FG disk both the Pwr and Exp form for fx-f boundary conditions have smaller radial stress compared to both the Pwr and Exp form for fr-f boundary conditions. For some specific values of $n$ and $m$ for all boundary conditions, it has been determined that the radial stress value increases along the radius of the FGM disk.

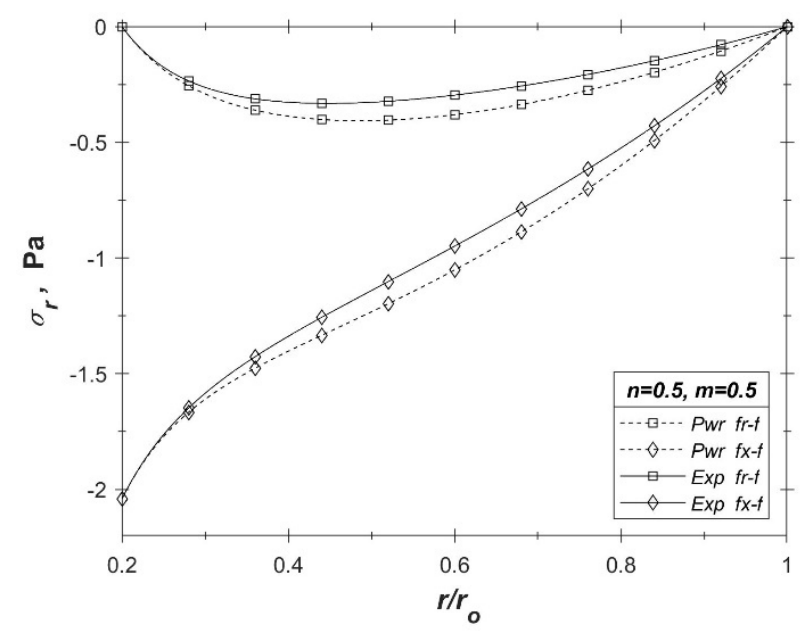

(a)

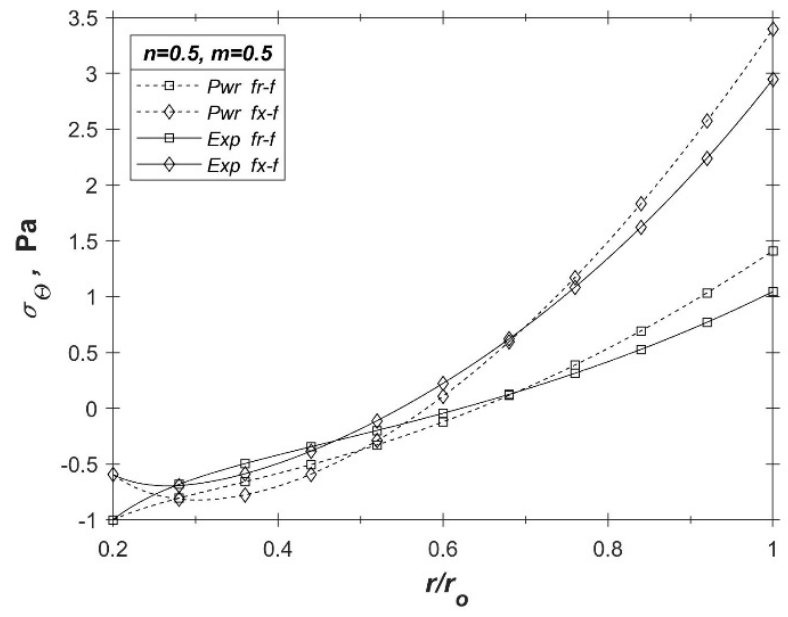

(b) 


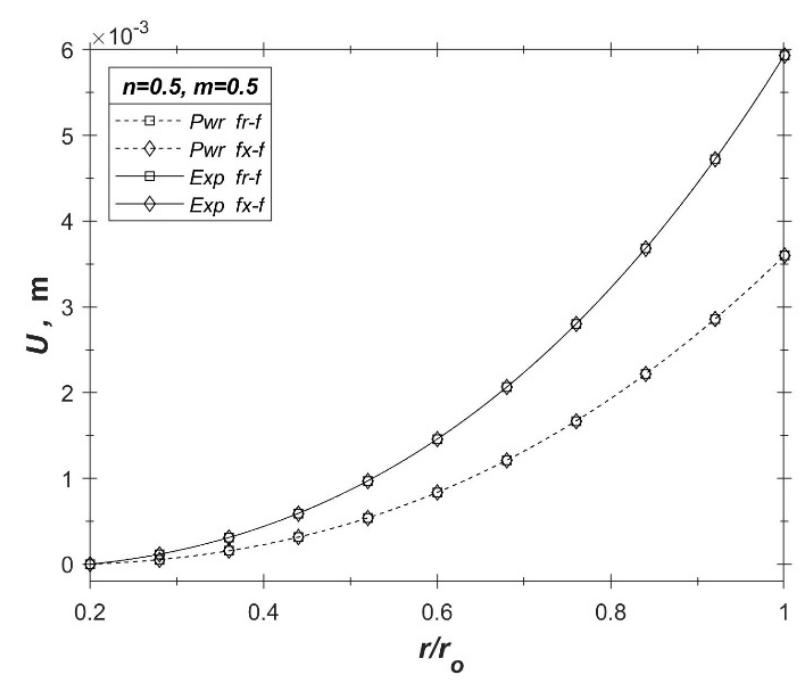

(c)

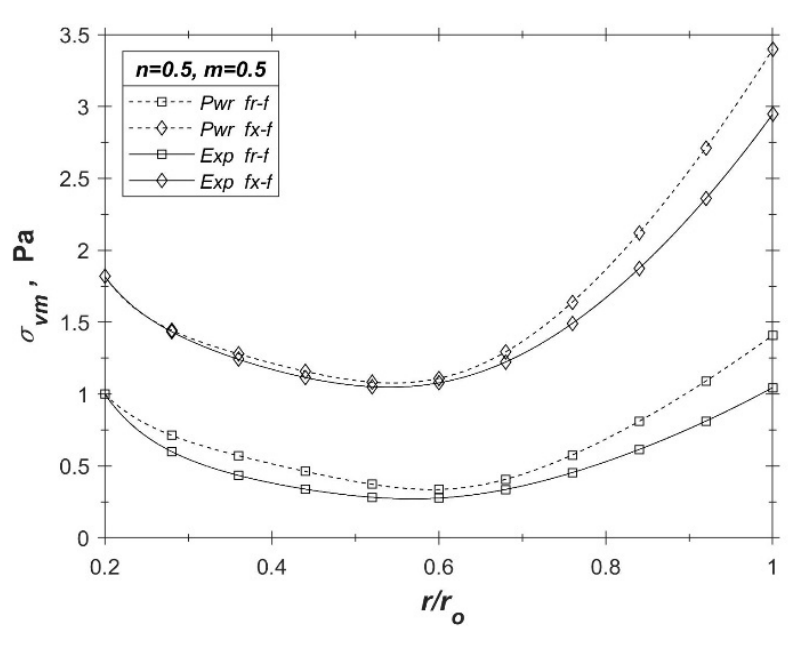

(d)

Fig. 1. Because of the effect of temperature with the material grading and boundary conditions on the distribution of stresses and displacement in FGM hollow disk (a) radial stress, (b) hoop stress, (c) radial displacement and (d) equivalent stress.

The hoop stress dispersion caused by thermal load up for FGM hollow disk along its radius for various $n$ and $m$ are produced in Figure 1-b. It is noticed that for the grading mark certain values $\mathrm{n}$ and $\mathrm{m}$ for Exp form with fr-f the highest hoop stresses may not happen at the outer side. $r / r_{0}$ increases in both forms and boundary conditions while the tangential stress value increases.

The displacement FGM hollow disk with fr-f and fx-f are shown in Figure 1-c for different gradient parameter values due to thermal load. It is observed that for some particular values of the grading mark $n$ and $m(n=0.5, m=0.5)$ and all boundary conditions the displacement increases for the FGM hollow disk along its radius.

Figure 1-d explain the equivalent stress respectively for various values of $\mathrm{n}$ and $m$, which are the inhomogeneity parameters by considering boundary conditions of both the Pwr and Exp model for material properties FG hollow disk along its radius due to thermal loading. It can be seen from Figure 1-d that for some particular values of the grading mark $n$ and $m$ for all boundary conditions, equivalent stresses values decrease to the midpoint of the thickness of the thick-walled disk, the rest increases

The displacement with radial, hoop, and equivalent stresses owing to the thermal loading up along the normalized radial direction for different values of $n$ in the hollow disc given in Figure 2. Thus, radial stresses (a) are high for the highest inhomogeneity parameter for both profiles. 


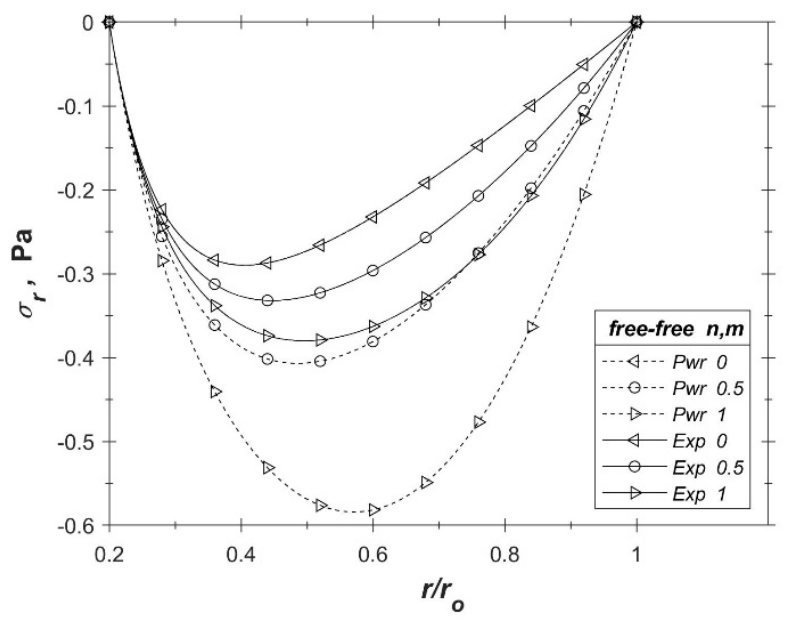

(a)

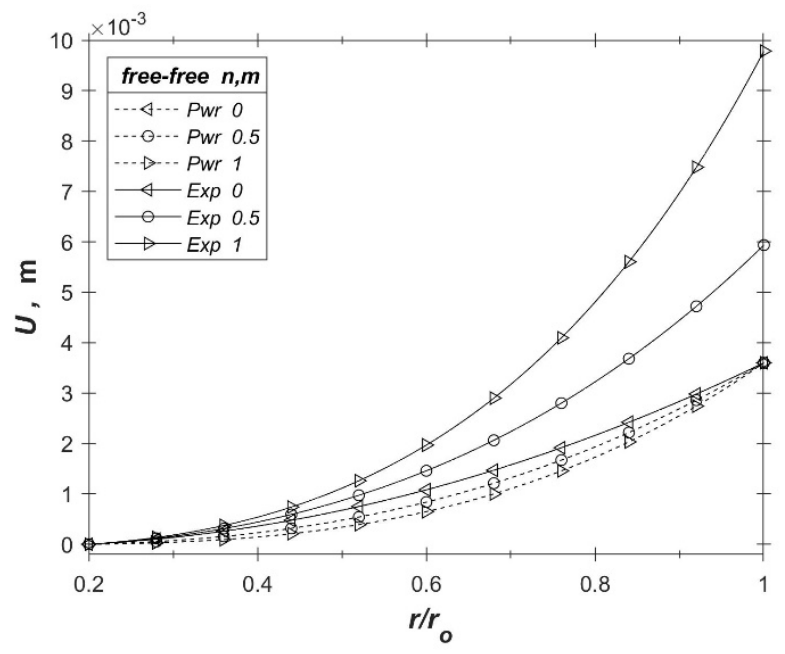

(c)

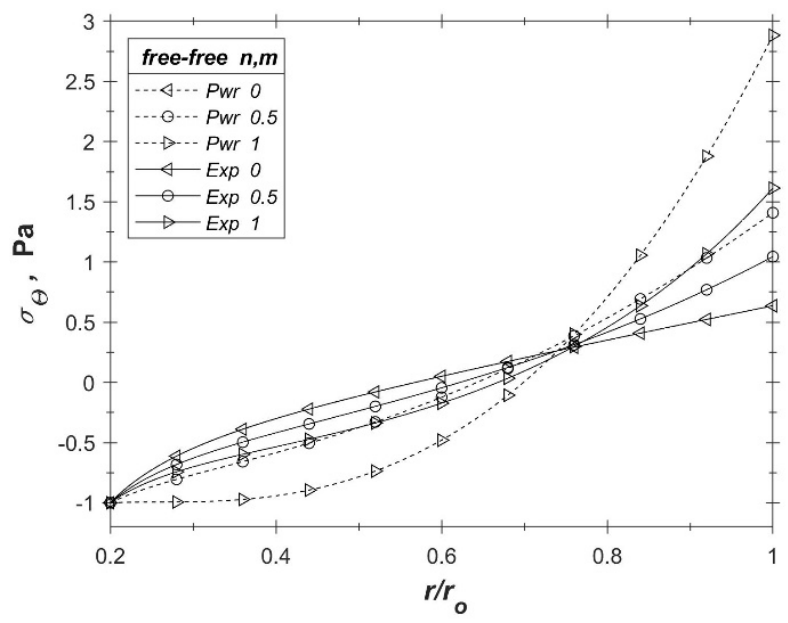

(b)

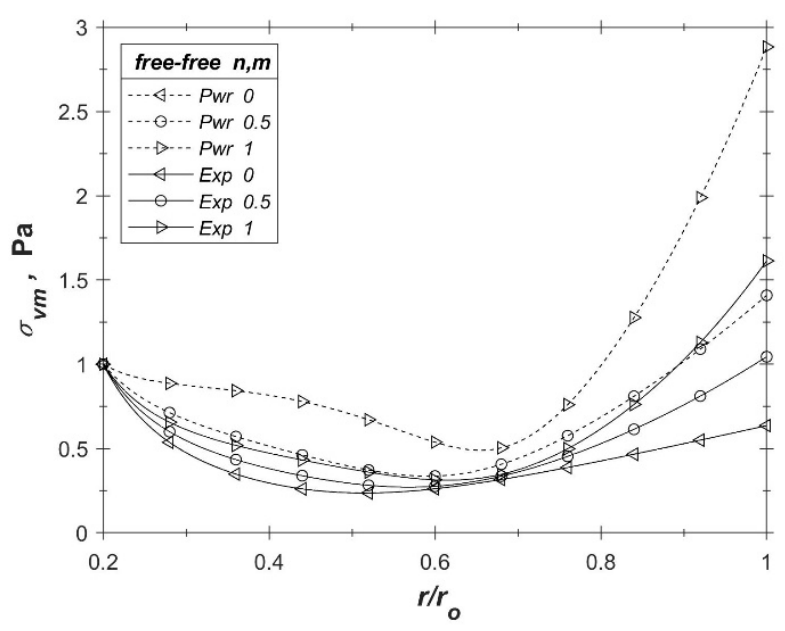

(d)

Fig. 2. Distribution of stresses and displacement in the hollow disk under the impact of temperature disk with the material grading and boundary conditions along the normalized radial direction for different values of $n, m$ (a) radial stress, (b) hoop stress, (c) radial displacement and (d) equivalent stress.

It was found that for $n$ and $m$ used for Pwr form with fr-f the maximum hoop stresses (in the figure b) may occur at the outside side. While the hoop stress values increase, increasing the $r / r_{0}$ for all boundary conditions and both Pwr and Exp function. The increase in gradient parameter for both the Exp profile and the Pwr profile of the FG disc findings in a reduction in the radial displacement (c) value. The radial displacement values increase, increasing the $r / r_{0}$ both Pwr and Exp function. As the gradient parameters increase, the equivalent stress (d) decreases down to the midpoint of the $r / r_{0}$ while the rest increases.

Figure 3 shows the radial displacement values for radial, tangential, and equivalent stresses caused by the thermal loading in the FG disk for various gradient parameter values and variable elasticity modulus and thermal expansion coefficient profiles. Looking at this figure, it is seen in the radial stress chart (a) that the stress reduces with increasing gradient parameter for both function form types, and the stress values increase with increasing thickness. 


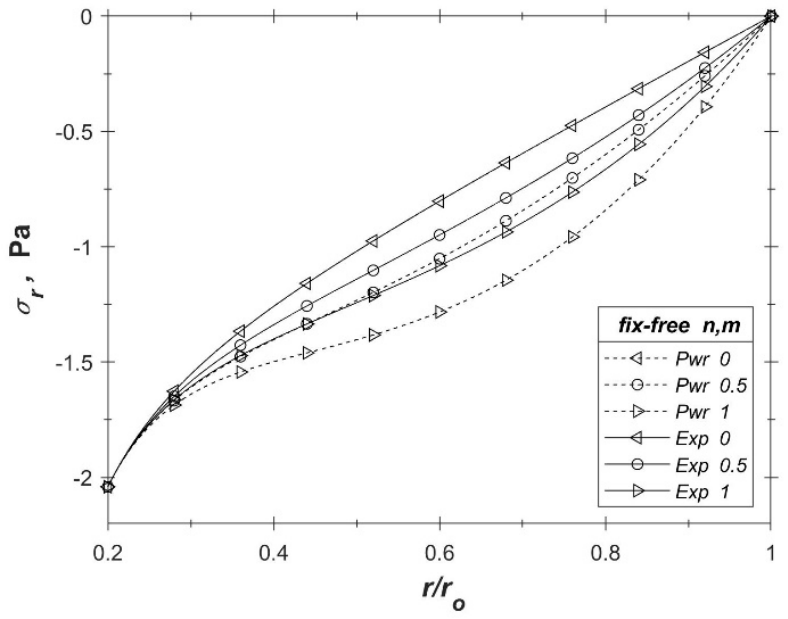

(a)

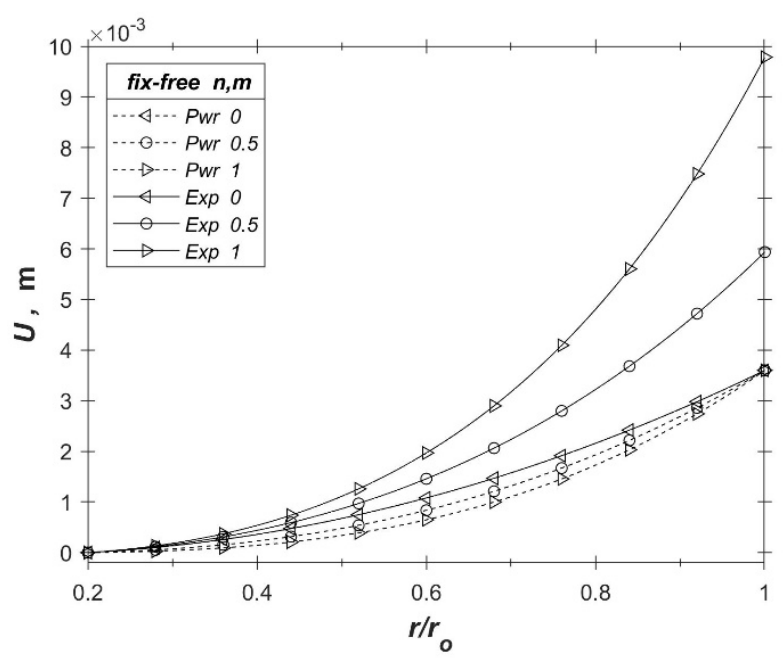

(c)

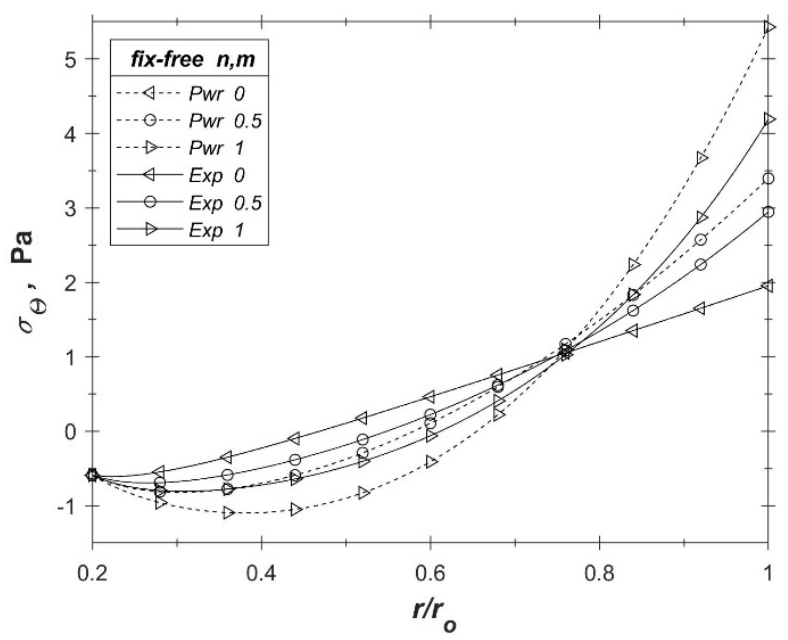

(b)

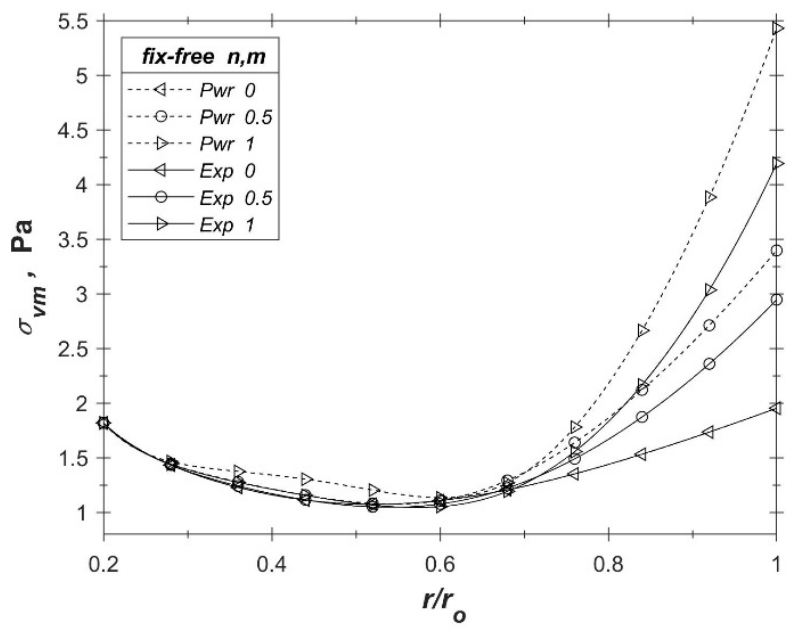

(d)

Fig. 3. In the hollow disk under the impact of temperature disk with the material grading and boundary conditions various values of the rating parameter $n, m$ (a) radial stress, (b) hoop stress, (c) radial displacement and (d) equivalent stress.

In the hoop stress graph (b), the tension value decreases with increasing gradient parameter for both profiles. However, the hoop stress values increase, increasing the $r / r_{0}$ for all boundary conditions and both Pwr and Exp. In the displacement graph (c), displacement values decrease with increasing gradient parameter for both profiles. The $r / r_{0}$ increases, and the displacement value increases for both profiles. In the equivalent tensile graph (d), as the gradient parameters increase, the equivalent stress (d) decreases down to the midpoint of the $r / r_{0}$ while the rest increases.

\section{Conclusions}

An examination of the hollow disk made of FGMs owing to thermo-mechanical loading is submitted. Thermo-elastic stresses are obtained for the hollow disk with both fr-f and fx-f boundary conditions. For FGM disk, modulus of elasticity and thermal growth coefficient are assumed to differ power-law and exponentially in radial way and numerical results are 
presented. When the numerical results in this study are evaluated, the results are briefly summarized below:

- The similar value of the $n, m$ radial pressure is highest when the hollow FGM disk, the powerlaw function profile and the exponential function profile disk are the lowest in fr-f and fx-f boundary conditions.

- When the radial stress distribution is examined, FGM is zero on the inner and outer surfaces of the disc, although the stress stays on as tension in the central portions for the free state.

- The hoop stress components linger stressed at the inner side of the functionally graded disc and compression at the outer side for the whole profiles and gradient parameters.

- The displacement components are lower on the inner edge of the FG disc and have higher values on the outer edge.

- The equivalent tensile component has tensile values at the inner edge of the FG disk and has an increased tensile value at the outer edge while decreasing towards the middle while constant for all profiles and gradient parameters.

- Differences in strain and displacement behavior of FG disk can be observed under fr-f and fxf boundary conditions.

- In certain applications, the influence of thermal loads is insignificant related to inertial forces but may be of equal or greater significance to others. With the solution method proposed in this study, it can help to get a solid idea for particular products. In addition, although FG disk gradient parameter is helpful before design, it can be said that it is an important parameter in determining stresses.

\section{References}

[1] Tutuncu, N. and B. Temel, A novel approach to stress analysis of pressurized FGM cylinders, disks and spheres. Composite Structures, 91, 385-390, 2009

[2] Jahromi, B.H., A. Ajdari, H. Nayeb-Hashemi, and A. Vaziri, Autofrettage of layered and functionally graded metal-ceramic composite vessels. Composite structures, 92, 18131822,2010

[3] Jahromi, B.H., G. Farrahi, M. Maleki, H. Nayeb-Hashemi, and A. Vaziri, Residual stresses in autofrettaged vessel made of functionally graded material. Engineering Structures, 31, 2930-2935, 2009

[4] Zhong, Z. and T. Yu, Analytical solution of a cantilever functionally graded beam. Composites Science and Technology, 67, 481-488, 2007

[5] Arefi, M., S. Firouzeh, E.M.-R. Bidgoli, and Ö. Civalek, Analysis of Porous Micro-plates Reinforced with FG-GNPs Based on Reddy plate Theory. Composite Structures, 112391, 2020 
[6] Civalek, Ö. and M. Avcar, Free vibration and buckling analyses of CNT reinforced laminated non-rectangular plates by discrete singular convolution method. Engineering with Computers, 1-33, 2020

[7] Hadji, L. and M. Avcar, Free Vibration Analysis of FG Porous Sandwich Plates under Various Boundary Conditions. 2020

[8] Jalaei, M. and Ö. Civalek, On dynamic instability of magnetically embedded viscoelastic porous FG nanobeam. International Journal of Engineering Science, 143, 14-32, 2019

[9] Zhang, J., S. Ullah, Y. Gao, M. Avcar, and O. Civalek, Analysis of orthotropic plates by the two-dimensional generalized FIT method. Computers and Concrete, 26, 421-427, 2020

[10] Arnab, B., S. Islam, A. Khalak, and A. Afsar, Finite difference solution to thermoelastic field in a thin circular FGM disk with a concentric hole. Procedia Engineering, 90, $193-$ 198,2014

[11] Zenkour, A., Stress distribution in rotating composite structures of functionally graded solid disks. Journal of Materials Processing Technology, 209, 3511-3517, 2009

[12] Çallığlu, H., M. Sayer, and E. Demir, Elastic-plastic stress analysis of rotating functionally graded discs. Thin-Walled Structures, 94, 38-44, 2015

[13] Sharma, J., D. Sharma, and S. Kumar, Vibration analysis of a rotating FGM thermoelastic axisymmetric circular disk using FEM. International Journal for Computational Methods in Engineering Science and Mechanics, 14, 262-270, 2013

[14] Durodola, J. and J. Adlington. Functionally graded material properties for disks and rotors. in Key Engineering Materials. 1997. Trans Tech Publ.

[15] Go, J., A. Afsar, and J. Song, Analysis of thermoelastic characteristics of a rotating FGM circular disk by finite element method. Advanced Composite Materials, 19, 197-213, 2010

[16] Hassani, A., M. Hojjati, E. Mahdavi, R. Alashti, and G. Farrahi, Thermo-mechanical analysis of rotating disks with non-uniform thickness and material properties. International Journal of Pressure Vessels and Piping, 98, 95-101, 2012

[17] Liew, K., S. Kitipornchai, X. Zhang, and C. Lim, Analysis of the thermal stress behaviour of functionally graded hollow circular cylinders. International Journal of Solids and Structures, 40, 2355-2380, 2003

[18] Kordkheili, S.H. and R. Naghdabadi, Thermoelastic analysis of a functionally graded rotating disk. Composite Structures, 79, 508-516, 2007

[19] Afsar, A. and J. Go, Finite element analysis of thermoelastic field in a rotating FGM circular disk. Applied Mathematical Modelling, 34, 3309-3320, 2010

[20] Peng, X.-1. and X.-f. Li, Thermoelastic analysis of functionally graded annulus with arbitrary gradient. Applied Mathematics and Mechanics, 30, 1211-1220, 2009

[21] Peng, X.-L. and X.-F. Li, Effects of gradient on stress distribution in rotating functionally graded solid disks. Journal of Mechanical Science and Technology, 26, 1483-1492, 2012 
[22] Naghdabadi, R. and S.H. Kordkheili, A finite element formulation for analysis of functionally graded plates and shells. Archive of applied mechanics, 74, 375-386, 2005

[23] You, L., Y. Tang, J. Zhang, and C. Zheng, Numerical analysis of elastic-plastic rotating disks with arbitrary variable thickness and density. International Journal of Solids and Structures, 37, 7809-7820, 2000

[24] Khorsand, M. and Y. Tang, Design functionally graded rotating disks under thermoelastic loads: Weight optimization. International Journal of Pressure Vessels and Piping, 161, 3340, 2018

[25] Aslan, T.A., A.R. Noori, and B. Temel, A unified approach for out-of-plane forced vibration of axially functionally graded circular rods. European Mechanical Science, 2, 3745,2018

[26] Celebi, K., D. Yarimpabuc, and N. Tutuncu, Free vibration analysis of functionally graded beams using complementary functions method. Archive of Applied Mechanics, 88, 729739,2018

[27] Celebi, K., D. Yarimpabuc, and T. Baran, Forced vibration analysis of inhomogeneous rods with non-uniform cross-section. Journal of Engineering Research, 6, 189-202, 2018

[28] Jing, L.-1., P.-j. Ming, W.-p. Zhang, L.-r. Fu, and Y.-p. Cao, Static and free vibration analysis of functionally graded beams by combination Timoshenko theory and finite volume method. Composite structures, 138, 192-213, 2016

[29] Khan, A.A., M. Naushad Alam, and M. Wajid, Finite element modelling for static and free vibration response of functionally graded beam. Latin American journal of solids and structures, 13, 690-714, 2016

[30] Roberts, S. and J. Shipman, Fundamental matrix and two-point boundary-value problems. Journal of Optimization Theory and Applications, 28, 77-88, 1979

[31] Agarwal, R., On the method of complementary functions for nonlinear boundary-value problems. Journal of Optimization Theory and Applications, 36, 139-144, 1982

[32] Yildirim, V., Free vibration analysis of non- cylindrical coil springs by combined use of the transfer matrix and the complementary functions methods. Communications in Numerical Methods in Engineering, 13, 487-494, 1997

[33] Celebi, K., D. Yarımpabuc, and I. Keles, A novel approach to thermal and mechanical stresses in a FGM cylinder with exponentially-varying properties. Journal of Theoretical and Applied Mechanics, 55, 2017

[34] Celebi, K., D. Yarimpabuc, and I. Keles, A unified method for stresses in FGM sphere with exponentially-varying properties. Structural Engineering and Mechanics, 57, 823835,2016

[35] Yıldırım, A., K. Celebi, and D. Yarımpabuç, A Practical Approach for Thermal Stress of Functionally Graded Annular Fin. Journal of Engineering Thermophysics, 28, 556-568, 2019 
[36] Yıldırım, A., D. Yarımpabuç, and K. Celebi, Thermal stress analysis of functionally graded annular fin. Journal of Thermal Stresses, 42, 440-451, 2019 